\title{
Protective Role of Gossypetin against Cyclophosphamide Toxicity in Human Lymphocyte Culture In vitro
}

\section{Ustunsoy $\mathbf{S}^{1 *}$, Akal $\mathrm{ZU}^{2}$ and Alpsoy $\mathrm{L}^{3}$}

${ }^{1}$ Medical Faculty, Medical Biochemistry Department, Fatih University, 34500, Istanbul, Turkey

${ }^{2}$ Science and Art Faculty, Biology Department, Fatih University, 34500, Istanbul, Turkey

${ }^{3}$ Medical Faculty, Medical Biology Department, Fatih University, 34500, Istanbul, Turkey

\begin{abstract}
Gossypetin is a flavonoid which has anti-mutagenic, anti-atherosclerotic, antioxidant, as well as cytoprotective and antimicrobial effects. The objective of this study was to investigate the cytoprotective role of gossypetin (GP) against cyclophosphamide (CP) toxicity in the human lymphocyte culture. Cytotoxic, necrotic and apoptotic effects of CP $(1 \mathrm{mM}), \mathrm{GP}(25,50$ and $100 \mu \mathrm{M})$ and combination of them (CP+GP) were studied by using MTT assay and Flow cytometry analysis. It was detected that CP significantly decreased cell viability rate via arresting cell cycle and increasing apoptosis/necroptosis. However, GP treatment reduced negative effects of $\mathrm{CP}$ at different concentrations. The most effective concentration of GP against CP toxicity was $25 \mu \mathrm{M}$. This concentration GP increased live cell number and cell viability, in addition decreased necrotic and late apoptotic cell quantity which were treated with $C P$. These results suggest that GP could attenuate the cytotoxic effects of CP and protect the healthy cells when it is used during chemotherapy.
\end{abstract}

Keywords: Cyclophosphamide; Gossypetin; Anticancer; Apoptosis; Necrosis; Cytotoxicity; Cell cycle

\section{Introduction}

Flavonoids are natural ingredients of plants and their by-products obtained by different methods like heating, boiling, fermenting, etc. They have been used as therapeutic solution to the health problems by humans for centuries even though they do not know the exact acting mechanism. Nowadays, there are approximately 4000 plant originated potentially therapeutic natural flavonoids in the market. They are classified into different groups according to their chemical structure [1]. These bioactive compounds have crucial roles at regulating important metabolic signaling pathways during physiological or pathophysiological cascades in human body [2,3]. Hence, there are hundreds of studies in the literature which have revealed the significance of these nutrients at both providing the healthy life and preventing/curing diseases such as atherosclerosis, diabetes, metabolic syndrome, neurodegenerative diseases and cancer by means of antiinflammatory or antioxidant properties. Moreover, flavonoids could increase or decrease the effect of key regulatory enzymes of metabolism via changing their expressions at DNA levels $[2,3]$.

Despite this huge number of flavonoids, quercetin is the most studied one due to its unique chemical and physiological properties in addition to broad prevalence in plants. Furthermore; luteolin, kaempferol, myricetin, catechin, rutin, fisetin, thymoquinone and gossypetin are the other remarkable bioactive compounds $[4,5]$. Gossypetin is usually extracted from the flowers of the hibiscus species. This molecule is named in chemistry as $3,5,7,8,3^{\prime}, 4^{\prime}$ hexahydroxy flavone. Previous studies revealed that gossypetin has anti-mutagenic, anti-atherosclerotic, antioxidant, cytoprotective and antimicrobial effects likewise quercetin and luteolin because of its unique chemical structure [6]. Ngai reported that gossypetin has antiproliferative effects on breast cancer (MCF-7) and hepatocellular cancer (HepG2) cell lines. Especially between 25 and $100 \mu \mathrm{M}$ GP is more effective on MCF-7 and HepG-2 [7]. In addition, a newly published paper has shown that gossypetin could protect cells against both radiation induced oxidative stress and radiation induced cellular damage via decreasing reactive oxygen species (ROSs) [8]. Previous studies have shown that ROSs make vital modifications on biomolecules like lipids, proteins and DNAs which change their morphology, biological functions, life spans and leads to chronic progressive diseases like atherosclerosis, cancer, inflammation, neurodegenerative diseases, and aging [9]. At this point, flavonoids have crucial importance because of their antioxidant properties. These bioactive substances can both decrease the production of reactive oxygen species and neutralize their toxic effects $[9,10]$. Moreover, Chen et al. have demonstrated another important effect of the gossypetin which inhibits LDL cholesterol oxidation, intracellular lipid accumulation and foam cell formation through intra cellular signaling molecules that have significant roles in the development of atherosclerosis such as PPRa, PPR $\gamma, A B C A 1$ and CD36 [11]. In addition, there are recently published papers suggesting new treatment regimens including antioxidant flavonoid molecules either for protecting the healthy cells against antineoplastic, cytotoxic, immunosuppressive agents or as a therapeutic agent alone [12].

Cyclophosphamide (CP) is a prodrug that is commonly used in the treatment of a broad spectrum of cancers and some sort of autoimmune diseases. Its mechanism of action depends on the active metabolite of 4-hyroxycyclophosphamide which is produced in the liver by cytochrome enzyme complexes [13,14]. In summary, this molecule adds an alkyl group to the DNA and prevents cellular replication. As a result, CP directs the cells to the apoptosis [13,14]. Although immunosuppression and cytotoxicity are unwanted side effects of $\mathrm{CP}$, these properties also make this drug a choice in the treatment of severe autoimmune diseases for reducing the disease burden and improving the patient's general health status [15].

In this study, we aimed to investigate the protective effects of GP against cellular damage which is formed by CP. As far as we know, this

*Corresponding author: Seyfettin Ustunsoy, Medical Faculty, Medica Biochemistry Department, Fatih University, 34500, Istanbul, Turkey, Tel: +90-2128663300/7021; E-mail: seyfettin.ustunsoy@fatih.edu.tr

Received January 25, 2016; Accepted February 17, 2016; Published February 22, 2016

Citation: Ustunsoy S, Akal ZU, Alpsoy L (2016) Protective Role of Gossypetin against Cyclophosphamide Toxicity in Human Lymphocyte Culture In vitro. Med chem 6: 088-092. doi:10.4172/2161-0444.1000330

Copyright: (c 2016 Ustunsoy S, et al. This is an open-access article distributed under the terms of the Creative Commons Attribution License, which permits unrestricted use, distribution, and reproduction in any medium, provided the original author and source are credited. 
is the first paper in the literature that is revealing the protective effect of the GP during CP chemotherapy.

\section{Materials and Methods}

All products used for cell culture studies (RPMI-1640, phytohaemaglutinin (PHA), fetal bovine serum (FBS), phosphate buffer saline (PBS), Ficoll, L-glutamine and penicillin-streptomycin (PS) were purchased from Biochrom AG (Mannheim, Germany) and Biological Industries (Kibbutz Beit Haemek, Israel). Cell proliferation assay kit (MTT) and Annexin-V CY3 Apoptosis Detection Kit Plus (containing SYTOX Green Dye) were purchased from Biovision (Mannheim, Germany), Ficoll-Hypaque, Propidium iodide (Sigma Aldrich, St Louis, Missouri, USA). Gossypetin is obtained from ChromaDex (Irvine, USA). All other chemicals used for cell culture studies and flow cytometry analysis were of reagent grade quality obtained from commercial sources.

\section{Lymphocyte isolation and culture of PMBC}

The peripheral blood mononuclear cells (PBMCs) were isolated from heparinized blood samples of a healthy volunteer person by centrifugation at $2500 \mathrm{rpm}$ for $25 \mathrm{~min}$ at room temperature according to the Ficoll-Hypaque density gradient method. All donors gave full written informed consent. The cloudy layer (containing thrombocyte, lymphocyte, monocyte, etc.) between the plasma and ficoll solution was separated with the help of a pipet. Lymphocytes were washed two times with PBS, and pellet was suspended in RPMI-1640 media. The number of viable PMBC cells was determined with the trypan blue method. Adjusted cell suspension density was equal to $1 \times 10^{6} \mathrm{cell} /$ $\mathrm{mL}$. The PBMCs were cultured on a 96 micro-well plate at a density of $1 \times 10^{6} \mathrm{cell} / \mathrm{mL}$ and incubated with $\mathrm{CP}(1 \mathrm{mM})$, GP $(25 \mu \mathrm{M}, 50 \mu \mathrm{M}$ and $100 \mu \mathrm{M})$ and $\mathrm{CP}(1 \mathrm{mM})+\mathrm{GP}(25,50$ and $100 \mu \mathrm{M})$ combinations supplemented with $2 \mathrm{mM} / \mathrm{l} \mathrm{L}$-glutamine, 10\% FBS and antibiotics (penicillin and streptomycin) for 4 days.

\section{MTT assay}

The cytotoxicity was measured by MTT assay. A cell viability test of GP was performed on cultured human lymphocytes to detect the maximal non-toxic concentration according to the manufacturer's protocol. A total of $1 \times 10^{4}$ cells were seeded on each well of a 96-well plate and cultured for 24 hours. The cells were treated with different concentrations of CP $(1 \mathrm{mM})$, GP $(25,50$ and $100 \mu \mathrm{M})$ and $\mathrm{CP}+\mathrm{GP}$ $(25,50$ and $100 \mu \mathrm{M})$ combinations in a serum-free medium for 24 and 48 hours. Gossypetin concentrations were selected according to previous studies [7]. At the end of the incubation process/time, the medium containing compound was exchanged with a fresh RPMI1640 medium, and $10 \mu \mathrm{L}$ of the reconstitute MTT mixture kit reagent were added to each well. After culturing for 4 hours, the absorbance of the sample was measured by using a microtiter plate reader (Bio-TEK) at $590 \mathrm{~nm}$. The viability of the cells was determined as the percentage of viable cells of the untreated control and experiments were performed in triplicate, and data are expressed as mean of triplicate measurements (mean $\pm \mathrm{SD}$ ) in percentage of untreated cells $(100 \%)$.

\section{Measuring apoptosis using flow cytometry}

Apoptotic and necrotic cells were identified with Annexin V-Cy3 and Sytox green according to the manufacturer's protocol, respectively. PMBC cells $\left(2 \times 10^{4}\right.$ cells/well $)$ were exposed to CP $(1 \mathrm{mM})$, GP $(25,50$ and $100 \mu \mathrm{M})$ and $\mathrm{CP}(1 \mathrm{mM})+\mathrm{GP}(25,50$ and $100 \mu \mathrm{M})$ combinations for $24 \mathrm{~h}$, after trypsinizaiton cells were collected and then washed with PBS, and incubated with $5 \mu \mathrm{l}$ Annexin V-Cy3 and $1 \mu \mathrm{l}$ Sytox green for 10 min in the dark. Cells incubated in the CP and GP free growth medium were used as the negative control. After staining a cell population with Annexin V-Cy3 and Sytox Green dye in the provided binding buffer, cell counting data were acquired BD Accuri C6 software. Apoptotic cells show red fluorescence (Cy3), dead cells show green fluorescence (sytotox green) and live cells show little or no fluorescence. These populations can be distinguished easily by flow cytometry detecting in the FL1 (FITC) channel (Ex. $488 \mathrm{~nm} / \mathrm{Em} .530 \mathrm{~nm}$ ) for Sytox Green dye and FL2 (PE) channel for Annexin V-Cy3 (Ex. 543 nm/Em. 570 nm).

\section{Cell cycle profiling with PI staining}

After trypsinization, cells were collected into $5 \mathrm{ml}$ propylene tubes $\left(1 \times 10^{6}\right)$ and centrifuged at 15,000 rpm, then stained with propidium iodide (PI) and analyzed by using BD Calibur flow cytometer, and data were evaluated with BD Accuri C6 software. In summary, cells were washed with $2 \mathrm{ml}$ PBS then centrifuged and supernatant was discarded. In addition, cells were fixed by adding $1 \mathrm{~mL}$ of $70 \%$ ice cold EtOH slowly on the pellet while vortexing to avoid cell clumping. Cells were incubated on ice for 30 minutes then centrifuged to get rid of the EtOH. Cell pellet was washed with PBS and centrifuged again. Supernatant was discarded and cell pellet was re-suspended in $1 \mathrm{~mL}$ of PI staining solution. Cells were incubated at $37^{\circ} \mathrm{C}$ for 40 minutes in PI staining solution and centrifuged again to remove the staining solution. Supernatant was discarded and the cell pellet was resuspended in $500 \mu \mathrm{l}$ PBS then analyzed at BD Calibur flow cytometer. All the centrifugations were carried out at $2000 \mathrm{~g}$ for 5 minutes.

\section{Statistical analysis}

All data are expressed as the mean \pm standard deviation (SD). Statistical differences between experimental groups were determined using Student's t-test. All statistical analyses were performed using GraphPad Prism 5 and SPSS 13.0. A value of $\mathrm{p}<0.05$ was considered statistically significant.

\section{Results}

\section{Gossypetin attenuate CP induced cytotoxicity}

In order to evaluate the cytoprotective effect of gossypetin we performed MTT cell viability assay. Human lymphocytes were incubated with different gossypetin concentrations and $1 \mathrm{mM} \mathrm{CP}$. Figure 1 presents MTT assay results. At $24^{\text {th }}$ hour, cells that were treated with $\mathrm{CP}$ showed significantly minimal cell viability rate when compared to the control group $(\mathrm{p}<0.05)$. In addition, GP100 concentration has a slight inhibitory effect on cell viability in human lymphocytes however GP25 concentration increased cell viability. Cell viability was $57.75 \%$ in CP group whereas in GP25, GP50 and GP100 was $130.74 \%, 86.20 \%$ and $81.89 \%$ respectively $(\mathrm{p}<0.05)$. When $\mathrm{CP}$ and different concentrations of GP were applied on lymphocyte culture, they decreased the cytotoxic effects of CP. Percentage of cell viabilities were $78.87 \%$ in CP+GP25, $79.74 \%$ in $\mathrm{CP}+\mathrm{GP} 50$ and $81.89 \%$ in $\mathrm{CP}+\mathrm{GP} 100$ group. MTT results showed us all GP concentrations have protective role on CP toxicity (Figure 1).

\section{Flow cytometric analysis of Annexin V on human lymphocytes}

The Annexin V binding assay is a universal, confidential, user friendly and fast flow cytometric technique that is used to discriminate apoptotic and necrotic cell death, the cells were stained with Annexin $\mathrm{V}-\mathrm{Cy} 3$ and Sytotox Green, and the time course study was performed using flow cytometry (Figure $2 \mathrm{a}$ and $2 \mathrm{~b}$ ). More than $39.9 \%$ of CPtreated cells showed Annexin V-negative/Sytotox green-positive staining at $2 \mathrm{~h}$, and approximately $16.6 \%$ of the cells were Annexin $\mathrm{V}$-positive/Sytotox green-negative. When the cells were treated with GP25 most of the cells remained double negative at $2 \mathrm{~h}$, moreover, 


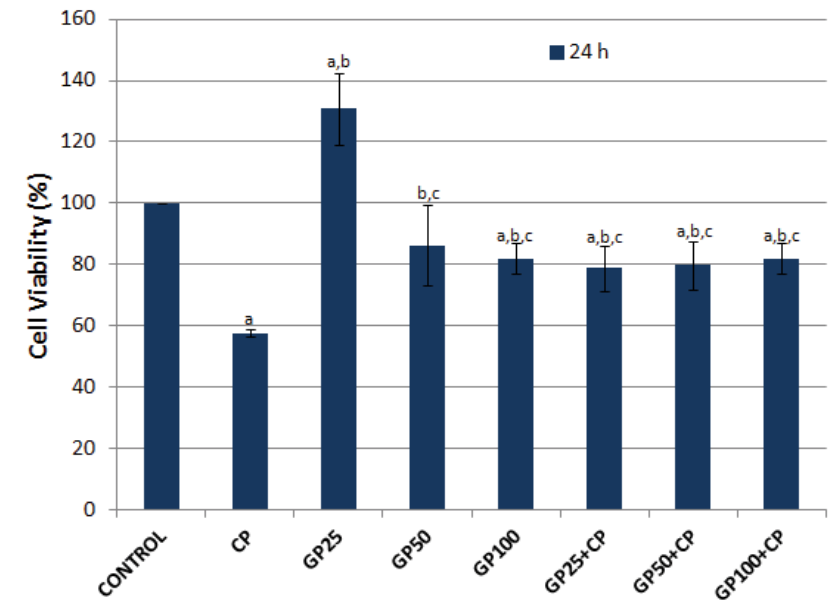

Figure 1: Cell viability rate of human lymphocyte after exposure to $\mathrm{CP}$, various concentrations of GP and CP+GP combinations. ${ }^{a} p<0.05$ compare with control group, ${ }^{b} p<0.05$ compare with CP group and ${ }^{c} p<0.05$ compare with $\mathrm{G} 25$ group.

GP25 increased the live cell number and decreased the number of necrotic and late apoptotic cells, suggesting that GP25 did not induce apoptosis at this concentration. In addition, $13.5 \%$ of CP+GP25 treated cells showed Annexin V-negative/Sytotox green positive staining at 2 h, and approximately $9 \%$ of the cells were Annexin V-positive/Sytotox green negative, and the percent of double negative cells were about $77 \%$. This means that GP25+CP significantly increased live cell number and decreased necrotic and apoptotic cells when compared with the $\mathrm{CP}$ treated group. GP50 treated cells showed 24\% Annexin V-negative/ Sytotox green positive staining at $2 \mathrm{~h}$, and approximately $15.2 \%$ of the cells were Annexin V-positive/Sytotox green positive, and the rest $60 \%$ were live cells. CP+GP50 treated group cells showed 21.9\% Annexin $\mathrm{V}$-negative/Sytotox green-positive staining at $2 \mathrm{~h}$, and approximately $25.4 \%$ of the cells were Annexin V-positive/Sytotox green negative. As a result, GP50+CP combination increased apoptotic cells and decreased necrotic cells when compared GP50 treated group. Last group GP100 treated cells showed $28.2 \%$ Annexin $\mathrm{V}$-negative/Sytotox green positive staining at $2 \mathrm{~h}$, and approximately $16.4 \%$ of the cells were Annexin $\mathrm{V}$-positive/Sytotox green negative. When the cells were treated with $\mathrm{CP}+\mathrm{GP} 100$ combination, cells showed 36.9\% Annexin V-negative/ Sytotox green positive staining at $2 \mathrm{~h}$, and approximately $7 \%$ of the cells were Annexin V-positive/Sytotox green negative. Our experimental data showed that GP100+CP increased necrotic cell and decreased apoptotic cells when compared with GP100 treated cells (Figure 3).

In summary, our flow cytometry assay results were complementary to the MTT results; CP decreased the live cell number, on the other hand GP25 increased the cell number near to the control group levels. However, GP50 and GP100 decreased live cell number slightly. Additionally, when we applied CP in combination with different GP concentrations on human lymphocytes, both live cell numbers increased and the percentages of apoptotic and necrotic cell decreased significantly when compared to the $\mathrm{CP}$ group. Also, our results demonstrated that $\mathrm{CP}$ has necrotic and apoptotic effects on human lymphocytes at $1 \mathrm{mM}$ concentration. According to the data presented in this article, the most effective concentration of GP was $25 \mu \mathrm{M}$ against CP toxicity (Figure 4 ).

\section{Flow cytometric analysis of cell cycle profiling with PI staining}

Intranucleosomal DNA breaks are important markers of cellular damage. Their presence means that apoptotic or necrotic common

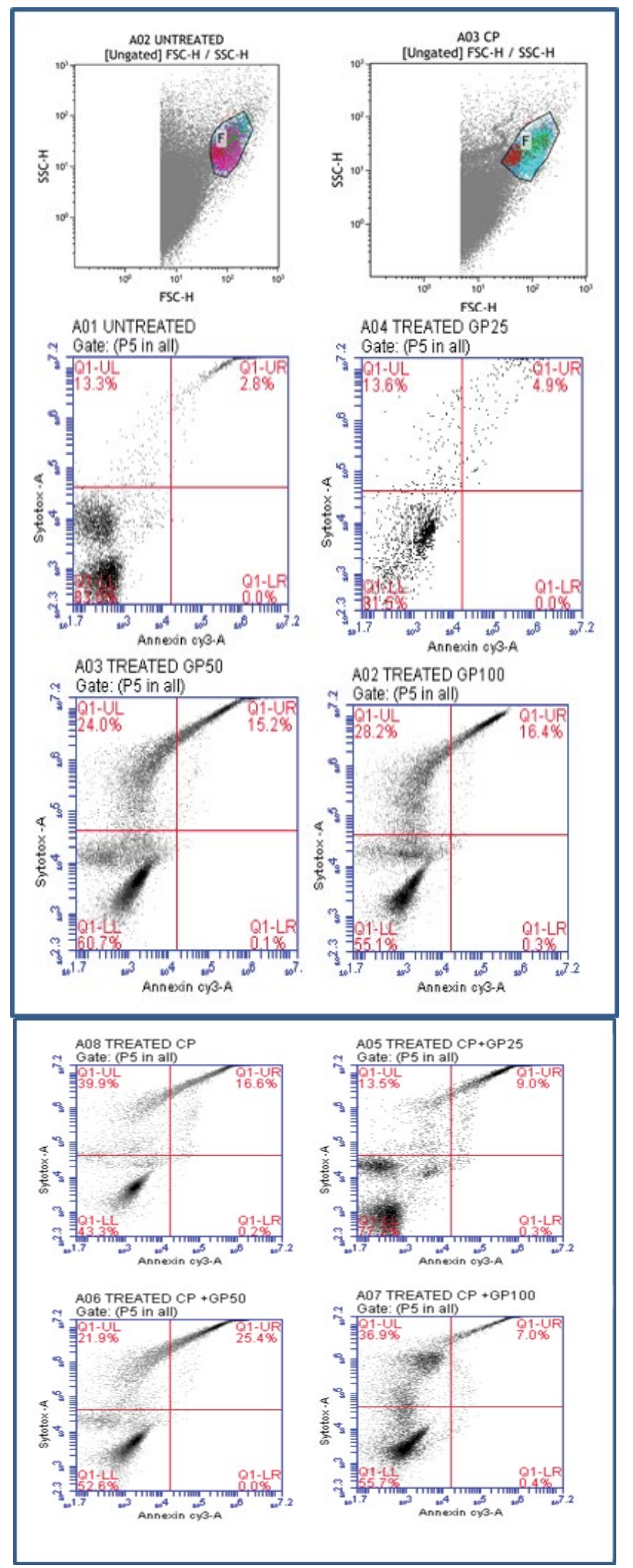

Figure 2a and b: Analysis of living, apoptotic and necrotic cell numbers by flow cytometer. 


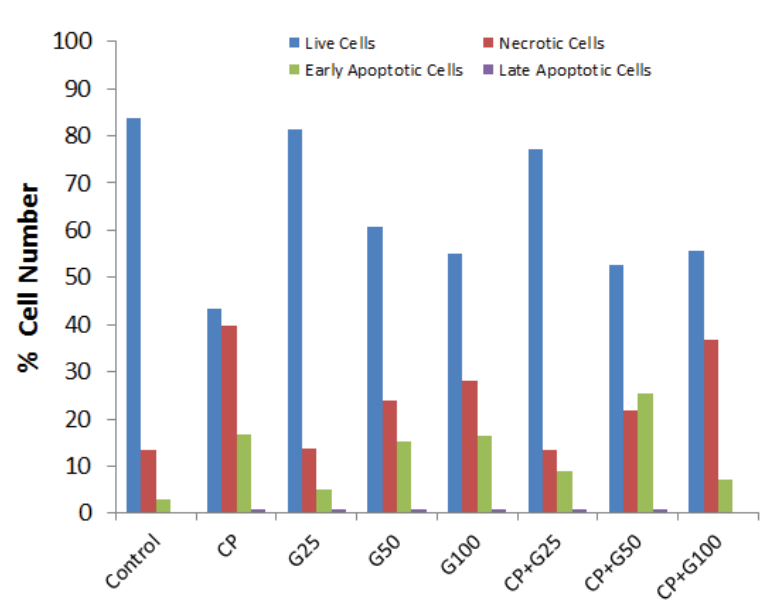

Figure 3: Percentages of live, necrotic and apoptotic cells in control and experiment groups analyzed by flow cytometer.

cascades takes place in the cell death arising from different causes. Cell cycle profiling with PI staining is a familiar, reliable, fast and easy flow cytometric method for the detection of DNA breaks, cell cycle arrest points and for the discrimination of viable, apoptotic or necrotic cells.

To determine whether the inhibitory effect of $\mathrm{CP}$ on human lymphocyte cell proliferation involved cell cycle changes, we examined cell cycle phase distribution of treated cells by flow cytometer. Our flow cytometric analysis of cell cycle profiling with PI staining revealed complementary data to the MTT assay results. According to this study results CP significantly inhibited cell cycle in G0/G1 phase. On the other hand, all of the applied gossypetin concentrations (GP25, GP50 and GP100) significantly induced cell viability but G25 was the most effective concentration among them. When GPs were applied with CP, they decreased the blocking effect of $\mathrm{CP}$ on cell cycle. As a result, all of the GPs have protected the cells against CP toxicity.

\section{Discussion}

$\mathrm{CP}$ is an antineoplastic prodrug belongs to the nitrogen mustard group which is converted to its active form in the liver. It is commonly used in the treatment of autoimmune diseases and various cancer types [16]. Although, CP takes place so often in cancer therapy regimens, like all other antineoplastic agents, may lead to new dysplasias which has the possibility of emergence of new cancer types during treatment because of its high toxic side effects. Thus, oncologists do not prefer to use CP alone, instead of that they combine it with other drugs, for example doxorubicin, to minimize the dosage for protecting normal healthy cells from CP toxicity [17-19]. Besides this, scientists have improved a new strategy against cytotoxic side effects of chemotherapeutic drugs which is adding flavonoids to the treatment regimens. Therefore, antineoplastic-immonosuppressive agents+flavonoid combinations are hot topic nowadays between researchers who are dealing with cancer therapy or chronic progressive diseases. $\mathrm{Xu}$ et al. have worked on ginsenoide $\mathrm{Rg} 3$ in combination with $\mathrm{CP}$ and revealed that this drug+flavonoid combination has prolonged the survival rate of mice by eliminating the drug's toxic side effects, and inhibiting angiogenesis via down regulating the VEGF (vascular endothelial growth factor) expression [20]. In addition to that, Sammy et al. have combined CP with luteolin and showed that their combination successfully decreased the tumor volume, however, prevented the weight loss which might be the sign of the healthy cell protection (one of the most common adverse

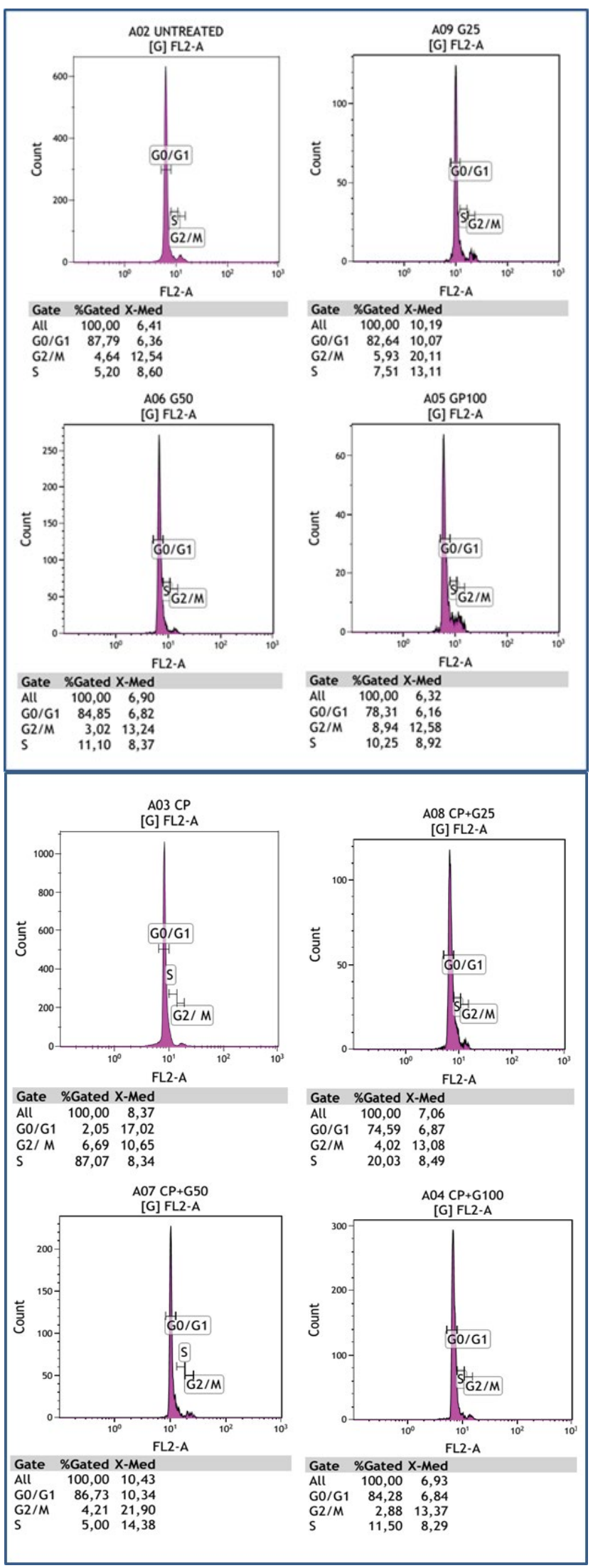

Figure 4a and b: Flow cytometric analysis in cell cycle progression between untreated and treated cultured human lymphocytes. 
effects of chemotherapy) [21]. Furthermore, resveratrol+CP mixture was tested in the treatment of breast cancer cells and found to be very effective at inhibiting cell proliferation through arresting cell cycle and leading them to the apoptosis [22]. Another study, which was designed by Alkan et al. demonstrated that curcumin $+\mathrm{CP}$ combination arrested breast cancer cell cycle at the G2/M phase, and led to cell cycle jamming at $\mathrm{S}$ phase. Moreover, the combination of curcumin and $\mathrm{CP}$ upregulated apoptotic Bax protein expression, however, downregulated antiapoptotic Bcl-2 protein expression. As a result, Bax/Bcl-2 balance was changed in favor of apoptosis and breast cancer cells were directed to the apoptosis by this way [23]. In the light of these findings, we thought that gossypetin too could be a good candidate for drug+flavonoid combinations strategy in cancer treatment for preserving healthy cells against drug caused cellular damage because of its high ROS scavenging capacity. Moreover, in this study, we have tested different GP and CP concentrations on the human lymphocytes in vitro and observed cell survival rates along with cell cycle proliferation phases. Consistent with other publications in the literature [24] our study confirmed that flavonoids are capable of protecting normal healthy cells against cytotoxic effects of chemotherapeutic agents like CP. According to our results, GP has significantly decreased cytotoxic effects of $\mathrm{CP}$ on human lymphocytes. While all applied GP concentrations were successful against CP damage, especially, the GP25 was the most effective dose at reducing $\mathrm{CP}$ toxicity. Furthermore, our flow cytometric analysis of cell cycle profiling with PI staining was consistent with cell viability results. CP arrested cell cycle in G0/G1 phase. On the other hand, gossypetin addition to the culture media increased cell viability close to the control group levels. Besides, GP25 increased cell viability via suppressing apoptotic and necrotic impacts of CP. When GPs were applied with CP, they decreased the blocking effect of $\mathrm{CP}$ on cell cycle. As a result, all of the GPs have protected the cells against CP toxicity. It means that, gossypetin fights against $\mathrm{CP}$ derived cellular damage through scavenging ROS, decreasing oxidative stress and supporting antioxidant mechanisms. Finally, it is important to mention that GP is a valuable and remarkable antioxidant biomolecule that could achieve to be a good partner in cancer chemotherapy like luteolin and quercetin.

\section{Conclusion}

In conclusion, as far as we know this is the first study in the literature which demonstrates that GP has cytoprotective activity against CP toxicity. The protective effects appear to be the result of the reduction of cytotoxicity via inhibition of ROS production and restoration of the antioxidant system. Although, we obtained promising results on the lymphocyte cell culture experiments, further in vitro cancer cell culture and animal studies are necessary for confirming whether the GP+CP complex is efficient in physiological conditions to become a potential new antitumour drug+flavanoid combination.

\section{References}

1. Ferreira CZ, Cária NZ, Victoriano G, Silva WF, Magalhaes JC (2014) Phytotherapy: an introduction to its history, use and application. Rev Bras PI Med 16: 290-298.

2. Magrone T, Perez de Heredia F, Jirillo E, Morabito G, Marcos A, et al. (2013) Functional foods and nutraceuticals as therapeutic tools for the treatment of diet-related diseases. Can J Physiol Pharmacol 91: 387-396.

3. Rios-Hoyo A, Cortes MJ, Rios-Ontiveros H, Meaney E, Ceballos G, et al. (2014) Obesity, Metabolic Syndrome, and Dietary Therapeutical Approaches with a Special Focus on Nutraceuticals (Polyphenols): A Mini-Review. Int J Vitam Nutr Res 84: 113-123.

4. Salvamani S, Gunasekaran B, Shaharuddin NA, Ahmad SA, Shukor MY (2014) Antiartherosclerotic effects of plant flavonoids. Biomed Res Int 2014: 480258.

5. Kleemann R, Verschuren L, Morrison M, Zadelaar S, van Erk MJ, et al. (2011) Anti-inflammatory, anti-proliferative and anti-atherosclerotic effects of quercetin in human in vitro and in vivo models. Atherosclerosis 218: 44-52.
6. Lin HH (2015) In Vitro and In Vivo Atheroprotective Effects of Gossypetin against Endothelial Cell Injury by Induction of Autophagy. Chem Res Toxicol 28: 202-215

7. Ngai LK (2006) Anti-proliferative activity of gossypetin. PhDThesis. The Chinese University of Hong Kong, Hong Kong, China.

8. Khan A, Manna K, Bose C, Sinha M, Das DK, et al. (2013) Gossypetin, a naturally occurring hexahydroxy flavone, ameliorates gamma radiationmediated DNA damage. Int J Radiat Biol 89: 965-975.

9. Halliwell B (2006) Reactive species and antioxidants. Redox biology is a fundamental theme of aerobic life. Plant Physiol 141: 312-322.

10. Cariddi LN, Sabini MC, Escobar FM, Montironi I, Manas F, et al. (2015) Polyphenols as possible bioprotectors against cytotoxicity and DNA damage induced by ochratoxin A. Environ Toxicol Pharmacol 39: 1008-1018.

11. Chen JH, Tsai CW, Wang CP, Lin HH (2013) Anti-atherosclerotic potential of gossypetin via inhibiting LDL oxidation and foam cell formation. Toxicol Appl Pharmacol 272: 313-324.

12. Sakthivel KM, Guruvayoorappan C (2015) Acacia ferruginea inhibits cyclophosphamide-induced immunosuppression and urotoxicity by modulating cytokines in mice. J Immunotoxicol 12: 154-163.

13. Huttunen KM, Raunio H, Rautio J (2011) Prodrugs - from serendipity to rational design. Pharmacol Rev 63: 750-771.

14. Hall AG, Tilby MJ (1992) Mechanisms of action of, and modes of resistance to alkylating agents used in the treatment of haematological malignancies. Blood Rev 6: 163-173.

15. Rosado MM, Diamanti AP, Capolunghi F, Carsetti R (2011) B cell modulation strategies in autoimmunity: the SLE example. Curr Pharm Des 17: 3155-3165.

16. Juma FD, Rogers HJ, Trounce JR (1980) The Pharmacokinetics of Cyclophosphamide, Phosphoramide Mustard and nor-Nitrogen Mustard Studied by Gas-Chromatography in Patients Receiving Cyclophosphamide Therapy. Brit J Clin Pharmaco 10: 327-335.

17. Trebunova M, Laputkova G, Slaba E, Lacjakova K, Verebova A (2012) Effects of docetaxel, doxorubicin and cyclophosphamide on human breast cancer cell line MCF-7. Anticancer Res 32: 2849-2854.

18. Klement G, Baruchel S, Rak J, Man S, Clark K, et al. (2000) Continuous lowdose therapy with vinblastine and VEGF receptor-2 antibody induces sustained tumor regression without overt toxicity. J Clin Invest 105: R15-24.

19. Hanahan D, Bergers G, Bergsland E (2000) Less is more, regularly: metronomic dosing of cytotoxic drugs can target tumor angiogenesis in mice. J Clin Invest 105: 1045-1047.

20. Xu TM, Xin Y, Cui MH, Jiang X, Gu LP (2007) Inhibitory effect of ginsenoside $\mathrm{Rg} 3$ combined with cyclophosphamide on growth and angiogenesis of ovarian cancer. Chin Med J (Engl) 120: 584-588.

21. Samy RP, Gopalakrishnakone P, Ignacimuthu S (2006) Anti-tumor promoting potential of luteolin against 7,12-dimethylbenz(a)anthracene-induced mammary tumors in rats. Chem Biol Interact 164: 1-14.

22. Singh N, Nigam M, Ranjan V, Sharma R, Balapure AK, et al. (2009) Caspase mediated enhanced apoptotic action of cyclophosphamide- and resveratroltreated MCF-7 cells. J Pharmacol Sci 109: 473-485.

23. Alkan FU, Anlas C, Cinar S, Yildirim F, Ustuner O, et al. (2014) Effects of curcumin in combination with cyclophosphamide on canine mammary tumour cell lines. Vet Med-Czech 59: 553-572.

24. Ghayomi F, Navaei-Nigjeh M, Baeeri M, Rezvanfar MA, Abdollahi M (2015) A mechanistic approach for modulation of chlorpyrifos-induced toxicity in human lymphocytes by melatonin, coenzyme Q10 and vinpocetine. Hum Exp Toxicol 0960327115607945. 